\title{
Caracterización de la endoscopia de vías digestivas altas en una población pediátrica colombiana
}

\section{Characterization of upper digestive tract endoscopy in a Colombian pediatric population}

\author{
Catalina González Áviles', Natalia Rojas Suárez², Aura Yaneth Córtes², \\ Christian Ernesto Melgar ${ }^{3}$
}

\begin{abstract}
Resumen
La endoscopia de vías digestivas altas en los pacientes pediátricos es una herramienta diagnóstica consulta que de bajo riesgo que ayuda a visualizar hallazgos y elementos importantes para la toma de decisiones terapéuticas. Objetivo: Caracterizar los hallazgos en los pacientes menores de 18 años que fueron sometidos a endoscopia digestiva alta, en un periodo de cuatro años, entre el 2011 a 2015, en dos centros de estudios de endoscopia de la ciudad de Neiva. Materiales y métodos: Estudio documental retrospectivo basado en los informes de estudios de endoscopia digestiva alta en pacientes menores de 18 años. Se analizó un total de 258 informes de pacientes, y los datos recopilados fueron género, edad, y diagnóstico endoscópico. Para el análisis de datos se utilizó el paquete estadístico SPSS versión 19.0 a través de estadística descriptiva. Resultados: De los 258 registros de endoscopia pertenecientes a pacientes entre los cero y 18 años, la distribución por genero fue: femenino $59.3 \%$ y masculino $39.5 \%$; la distribución por grupos etarios fue: escolares $40 \%$, preescolares $26 \%$, adolescentes $21 \%$ y lactantes $13 \%$, los diagnósticos endoscópicos de mayor frecuencia fueron: esofagitis preescolares $(8,9 \%, n: 6)$, gastritis en $79 \%$ ( $n$ : 203) del total de pacientes, de los cuales el $50,2 \%$ ( $n: 102)$ fue gastritis crónica, y se tomaron biopsias en el $77 \%$ de los casos. Conclusión: Los hallazgos endoscópicos en la población pediátrica evidencian esofagitis con mayor frecuencia en pre escolares y escolares en relación con múltiples factores; la gastritis crónica o folicular se presenta hasta en el $80 \%$ de los pacientes, la sedación se utilizó en el $100 \%$ de los pacientes, y en la mayoría de los casos, fue realizada por no anestesiólogos.

Palabras clave: Endoscopia, pediatría, esofagitis, gastritis, cuerpo extraño.
\end{abstract}

\begin{abstract}
Upper digestive tract endoscopy in pediatric patients is a low-risk diagnostic tool that helps to visualize findings and important elements for therapeutic decision making. Objective: To characterize the findings in patients under 18 years old who underwent upper gastrointestinal endoscopy, in a period of four years, between 2011 and 2015, at two endoscopy centers in the city of Neiva. Materials and methods: It is a documentary retrospective study, based to reports of upper gastrointestinal endoscopy studies. It was performed in children under 18 years in database of two endoscopy centers at Neiva (Colombia). The study was carried out between from January 2011 to January 2015, We documented 258 patients and data such as gender, age, endoscopic diagnosis were obtained. Data analysis was done by using SPSS version 19.0 through descriptive statistics. Results: 258 records endoscopies between zero and 18 years
\end{abstract}

1 Médica. Universidad Surcolombiana. Hospital Universitario Hernando Moncaleano Perdomo.

2 Médica Interna. Universidad Juan N. Corpas. Hospital Universitario Hernando Moncaleano Perdomo.

3 Médico Internista, Docente Universidad Surcolombiana, Gastroenterólogo Universidad Nacional de Colombia. Hospital Universitario Hernando Moncaleano Perdomo.

* Correspondencia: Catalina González Áviles. Correo electrónico: Email: catalinagonzalezaviles@gmail.com Recibido: 29/07/2016. Revisado: 15/09/2016. Aceptado: 6/12/2016 
were found, the distribution by gender was female with $59.3 \%$ and female $39.5 \%$, the distribution by age group was: kids $40 \%$, followed by toddlers $26 \%$, teenagers $21 \%$ and babies $13 \%$. endoscopic diagnoses more frequent were esophagitis in toddlers $(8,9 \%, n=6)$. Gastritis occurred in $79 \%(n=203)$ of all patients, chronic gastritis were $50,2 \%(n=102)$ of them. Biopsies were taken in $77 \%$ of all cases. Conclusions: Endoscopic findings in pediatric population showed that esophagitis was presented more frecuently in kids and toddlers, it could be related to multiple factors. Chronic gastritis was presented in $80 \%$ of patients and sedation was used in $100 \%$ of patients, It cases was performed most often by nonanesthesiologists.

Key words: Endoscopy, Pediatrics,Esophagitis, Gastritis, Foreing Bodies

\section{Introducción}

Entre el $2 \%$ y $4 \%$ de todas las consultas pediátricas, se deben a trastornos gastrointestinales funcionales, y, el 10\% de estos pacientes presenta dolor abdominal recurrente ${ }^{[1]}$; la patología de los pacientes pediátricos es amplia y ligada a múltiples noxas, lo que genera el uso de múltiples ayudas diagnósticas ${ }^{[2]}$, la endoscopia de vías digestivas altas es una herramienta diagnóstica terapéutica de gran utilidad y con amplio uso en la población menor de 18 años ${ }^{[3]}$. Siendo además una exploración simple y con bajos riesgos que permite el adecuado manejo de la enfermedad gastrointestinal ${ }^{[4]}$.Los trabajos de endoscopias digestivas altas realizadas en niños muestran distintos hallazgos: extracción de cuerpo extraño (ECE), hernia de hiato $(\mathrm{HH})$ y/o esofagitis distal, lesiones por cáusticos, hemorragia digestiva alta (HDA) y la gastrostomía endoscópica percutánea (PEG) ${ }^{[5]}$.

También se ha investigado la indicación de la endoscopia de vías digestivas altas en los pacientes menores de 18 años [6] donde se concluye la necesidad de evaluar críticamente la indicación de los procedimientos, además de la frecuencia de uso de la sedación en las endoscopias en niños [7].

En este estudio se llevó a cabo la caracterización de los hallazgos en los pacientes menores de 18 años, a quienes se les realizó endoscopia digestiva alta en un periodo de cuatro años, de 2011 a 2015 en dos centros de estudios de endoscopia de la ciudad de Neiva.

\section{Materiales y métodos}

Se realizó un estudio documental retrospectivo con la revisión de los reportes de endoscopia digestiva en menores de 18 años desde enero de 2011 hasta enero de 2015 en dos centros de endoscopia en la ciudad de Neiva, uno de la red pública y otro de la red privada de atención. Antes de la revisión documental se recibió el aval de los comités de ética de las instituciones involucradas en el estudio.

Las endoscopias digestivas en menores de 18 años, registradas en los reportes de centros de estudios, fueron realizadas con gastroscopios GIF-H180 (Olympus) de 9,8 mm de diámetro externo y con un canal de trabajo de $2,8 \mathrm{~mm}$. Durante la realización de las endoscopias digestivas siempre se registró presencia de gastroenterólogos o cirujanos pediatras con entrenamiento en procedimientos endoscópicos, acompañados de dos enfermeras con entrenamiento en endoscopias.
También se reportó que la preparación de los pacientes para el estudio consistía en ayuno mínimo de 8 horas; los pacientes recibieron sedación por médicos anestesiólogos y no anestesiólogos siempre con un acceso venoso confiable y con anestesia faríngea, previa explicación del procedimiento y firma de consentimiento informado por familiares. Los reportes fueron validados de acuerdo al formato de recolección de datos, de modo que se logró un adecuado coeficiente de correlación. Respecto a las descripciones de las variables se tuvo en cuenta el atlas de endoscopia Tadataka Yamada. El análisis estadístico se realizó con SPSS 19.0. Las variables analizadas fueron número de endoscopias realizadas, el número de pacientes sometidos a dicha exploración, el género y la edad de los mismos, la sedación empleada y variables relacionadas entre ellas.

\section{Resultados}

Se estudió una población pediátrica de 258 pacientes entre los 0 y 18 años, registrada desde el año 2011 hasta el año 2015, que se distribuyó en grupos etarios de la siguiente manera: lactante ( 1 mes a 2 años), preescolar (2 años a 5 años), escolar (6 años a 11 años), adolescentes (12 años a 18 años). La mayoría de la población se determinó en el grupo de escolares con un $40 \%$, seguido de preescolares $26 \%$, adolescentes $21 \%$ y lactantes $13 \%$. La distribución de procedimientos endoscópicos por género fue $59.3 \%$ femenino y $39.5 \%$ masculino.

La sedación se administró en un $75.2 \%$ por médicos no anestesiólogos y $24.8 \%$ por anestesiólogos. Las patologías presentadas, según hallazgos endoscópicos por grupo etario fueron: esofagitis en preescolares $8.9 \%$ (n: 6) y escolares $4.8 \%$ (n: 5). El compromiso más severo, según la clasificación de los Ángeles, se presentó en este mismo grupo etario (Grado D). La gastritis se presentó en el 79\% (n : 203) del total de pacientes, de estos casos, el 50.2\% (n : 102) fue gastritis crónica, el 11.3\% ( $\mathrm{n}: 23$ ) fue gastritis erosiva, el 38.4\% (n : 78) fue gastritis folicular (tabla 1). El compromiso por gastritis fue mayor en mujeres con una diferencia de $20.8 \%$ en crónica y $30.4 \%$ en folicular.

La hernia hiatal solo se presentó en el 1, 85\% (n: 1) de los adolescentes. De los pacientes que presentaron cuerpo extraño, el 52\% fueron lactantes y el $64,7 \%$ de sexo femenino (tabla 2 ). Del total de los procedimientos, el 59, 3\% aplicó al género femenino; y el $77 \%$ de los casos fueron remitidos a estudio histológico. 
R.F.S Revista Facultad de Salud

Julio - Diciembre de 2016;8(2):26-29
Caracterización de la endoscopia de vías digestivas altas en una población pediátrica colombiana

\section{Discusión}

La endoscopia de vías digestivas altas en menores de 18 años representa gran utilidad en la práctica clínica y aporta hallazgos fundamentales en el abordaje de este grupo poblacional. Recientemente, se han publicado múltiples estudios que documentan los cambios en los hallazgos endoscópicos en menores de 18 años en poblaciones, tradicionalmente, sin cambios endoscópicos relevantes en este grupo etario ${ }^{[7]}$.

En el presente estudio se caracterizaron los hallazgos endoscópicos de una población de pediátrica ( 0 a 18 años) en la región sur colombiana, frente a la etiología, según reporte endoscópico, la mayoría de pacientes con esofagitis se presentó en el grupo de pre escolares y escolares $(13,75 \%)$ [8]; Es de anotar que aunque la clasificación de los Ángeles no está validada aún para la población pediátrica puede ser muy útil en la evaluación de la severidad de la esofagitis en niños de 36 meses a 12 años ${ }^{[9]}$. El mayor compromiso dentro de este grupo etario, está relacionado con un alto componente atópico dentro de los padres y los pacientes en los que se ha documentado este fenómeno, el uso continuo de biberones, el subsecuente daño dental y fenómenos medioambientales como la exposición al tabaco ${ }^{[10][11]}$.

En el grupo de pacientes con gastritis la mayoría presentó el diagnóstico endoscópico de crónica, que puede estar ligada con la alta prevalencia de helicobacter pylori en la población estudiada. La suma de la gastritis crónica y folicular se presentó en más del $80 \%$ de los reportes estudiados, lo cual es concordante con las publicaciones que documentan la presencia del H. Pylori en poblaciones menores de 18 años ${ }^{[12]}$ generando que este estudio sea una puerta para la investigación de la relación endoscópica con la histología en nuestra población.

La toma de biopsias en los estudios endoscópicos son cada vez más importantes tanto para documentar presencia de helicobacter pylori como para determinar la posibilidad de enfermedades concomitantes ${ }^{[13]}$, en esta revisión más del $75 \%$ de los casos se tomaron biopsias sin reportar complicaciones secundarias.

Los cuerpos extraños son un motivo de consulta frecuente en los servicios de urgencias tanto de pediatría como en adultos, se documentó en el $75 \%$ de los casos en menores de 5 años hallazgo similar al publicado en distintas series del mundo [14] [15], la relación entre hombres y mujeres en esta serie de casos evidencia mayor compromiso en el sexo femenino distinto a grandes series mundiales en las que no hay un franco predominio por género, esta situación puede ser atribuible al número de endoscopias realizadas a niñas.

Los procedimientos documentados en el presente estudio requirieron sedación en todos los casos contrario a otros datos donde se evidenció un menor número de sedaciones en esta población ${ }^{[1]}$, la gran mayoría de sedaciones fueron realizadas por médicos no anestesiólogos, congruente con literatura publicada en nuestro país que respalda este procedimiento por no anestesiólogos en casos específicos (ASA I - II) ${ }^{[16]}$.
La endoscopia digestiva representa una herramienta útil en menores de 18 años en donde se hallan elementos importantes para la toma de decisiones terapéuticas, además de permitir el abordaje histológico bajo condiciones de escasas complicaciones.

\section{Bibliografía}

1. M.A Gómez, O.F. Ruiz, J. Riveros. Utilidad de la endoscopia digestiva alta diagnóstica en pacientes menores de 18 años. Rev Colom Gastro. 2014; 29 (2): 112-16.

2. Sheiko MA, Feinstein JA, Capocelli KE, Kramer RE. The concordance of endoscopic and histologic findings of 1000 Pediatric EGDs. Gastrointest Endosc. 2015; 81(6): 1385-1391.

3. Fox VL. Pediatric endoscopy. Gastro Endos Clinics Of North America. 2000; 10: 175-94.

4. Zahavi I, Arnon R, Ovadia B, et al. Upper gastrointestinal endoscopy in pediatric patient. Israel Journal of Medical Science. 1994; 30: 664-67.

5. Gómez, J. Barrio, L. Izquierdo et al. Gómez J, Barrio J, Izquierdo R, Simón $G$. Estudio retrospectivo sobre la endoscopia pediátrica desarrollada en un servicio de endoscopias de adultos. Rev Esp Enf Dig. 2010; 102 (2):100-07.

6. M.A Gómez, O.F. Ruiz, J. Riveros. Utilidad de la endoscopia digestiva alta diagnóstica en pacientes menores de 18 años. Rev Colom Gastro, 2014; 29 (2): 112-16.

7. Carvalho MA, Machado NC, Ortolan EV, Rodrigues MA. Upper Gastrointestinal Histopathological Findings in Children and Adolescents With Nonulcer Dyspepsia With Helicobacter Pylori Infection. J Pediat Gastr Nutr. 2012; 55 (5): 523-529.

8. Campanozzi A, Boccia G, Pensabene L. Prevalence and Natural History of Gastroesophageal Reflux: Pediatric Prospective Survey, Pediatrics 2009; 123 (3): 779-83.

9. Tolia V, Gilger MA, Barker PN, Illueca M. Healing of Erosive Esophagitis and Improvement of Symptoms of Gastroesophageal Reflux Disease After Esomeprazole Treatment in Children 12 to 36 Months Old. J Pediatric Gastr and Nutr. 2015; 60 (7): S31-6.

10. Fildes $A$, van Jaarsveld $C H$, Llewellyn $C$, Wardle J, Fisher A. Parental Control Over Feeding in Infancy. Influence of Infant weight, appetite and feeding method. Appetite 2015; 91: 101-06.

11. Martonffy Al. Oral Health: Prevention of Dental Disease. FP Essent. 2015; 428: 11-5.

12. Kalach N, Papadapoulos S., Asmar E. In French Children, Primary Gastritis Is More Frequent Than Helicobacter Pylori Gastritis. Dig Dis Sci. 2009; 54 (9): 1958-1965.

13. Serrano CA, Toledo H, et al. Iron Deficiency and IL lâ Polymorphisms in Helicobacter pylori-infected Children. Helicobacter. 2016; 21 (2): 124-30. 
14. Kramer RG. Lerner DG, Lin T, et al. Management of Ingested Foreign Bodies in Children: A Clinical Report of the NASPGHAN Endoscopy Committee. J Pediatr Gastr Nutr. 2015; 60 (4): 562-74.

15. Muñoz MP, Maluje R, Saitua F. Cuerpo Extraño Gastrointestinal en Niños. Rev Chil Pediatr 2014; 85 (6): 682-89
16. Forero EA. NAAP: Sedación profunda con propofol para endoscopia digestiva administrada por no anestesiólogos. Posición del endoscopista en el 2011. Rev Col Gastroenterol. 2011; 26 (2): 107-16. 